\title{
OPTICAL MULTI-SENSOR METROLOGY FOR EXTRUDED PROFILES
}

\author{
Albert Weckenmann, Johannes Bernstein
}

University Erlangen-Nuremberg, Chair Quality Management and Manufacturing Metrology, Naegelsbachstr. 25, 91052 Erlangen, Germany, (Albert.Weckenmann@qfm.uni-erlangen.de, $₫$ bernstein@qfm.uni-erlangen.de, +499131852 6545)

\begin{abstract}
Nowadays the process-control of concave extruded profiles is a measuring task with rising requirements. A novel optical bi-sensorial measurement system - consisting of a shadow- and a light-section-system - as well as suitable methods of analysis for in-line inspection are presented. The proposals help to ensure the product quality on a higher level than before. The combination of dimensional accuracy and data-density leads to excellent results. Extruded profiles are semi-finished products (from steel, brass, aluminum, synthetics...) which are appointed for wide applications in technical products. For monitoring and controlling the parts in-line adequately, today often the shading technology is used. It detects - neglecting the profiles coat in measurement range - with high precision the dimension of the objects shadow orthographically to the axis of illumination. As a matter of fact, concave and local areas cannot be recorded. Alternatively, light-section-systems can measure concave zones and undercut zones but do not comply with the required accuracy. The combination of accuracy and data-density qualifies the mentioned multi-sensor metrology for realizing a maximum of efficiency in process control what ensures product quality and less defective goods. The optical multi-sensor measurement system has to be calibrated and aligned to detect the same surface zone despite of high refresh rates and optical resolutions. The metered characteristics will be coordinate transformed to extrinsic world-coordinates for evaluating form deviations of complex parts. An appropriate user-interface enables to re-calculate measurement objects in-line and evaluate the conformity of the part consequently. Finally the real length information assists to influence the process control. After a successful test in the laboratory the results will be proved in production to the target: measurement uncertainty of better than $0.1 \mathrm{~mm}$ at every profile.
\end{abstract}

Keywords: multi-sensor-measurement, optical measurement, laser scanning.

(C) 2010 Polish Academy of Sciences. All rights reserved

\section{Introduction}

Optical non-contact manufacturing metrology with high speed qualifies for different materials and ambient environments. Unfortunately, the present optical sensor systems cannot deal with all performances the customer of the parts demands for. Extruded profiles are semifinished products which are appointed for wide applications in manufacturing of technical products. Nowadays-used optical sensors in process control working to the shading technology detect the objects' shadow orthographically to the axis of illumination. Often manufactured objects in production are hot, afflicted with oxide scale and move inaccurately in processes. In order to rework the profile to a final product, the customer (using the semifinished profiles) demands great accuracy at every point of the profiles outline contour. On the one hand shadow systems are very accurate but unable to measure concave profiles. They serve only with two-point information of the objects' outline with one diameter. They reach rapid refresh rates of more than $500 \mathrm{~Hz}$ and resolutions of $0.01 \mathrm{~mm}$ [1]. On the other hand light-section systems (as well state of the art) can measure concave profiles zones fundamentally, but not with the required accuracies of better than $0.1 \mathrm{~mm}$. Their refresh rate exceeds rarely few $\mathrm{Hz}$ what can be too short for in-line applications. An optical multi-sensor measurement system - combining either - leads to better measurement results, see Fig. 1. 


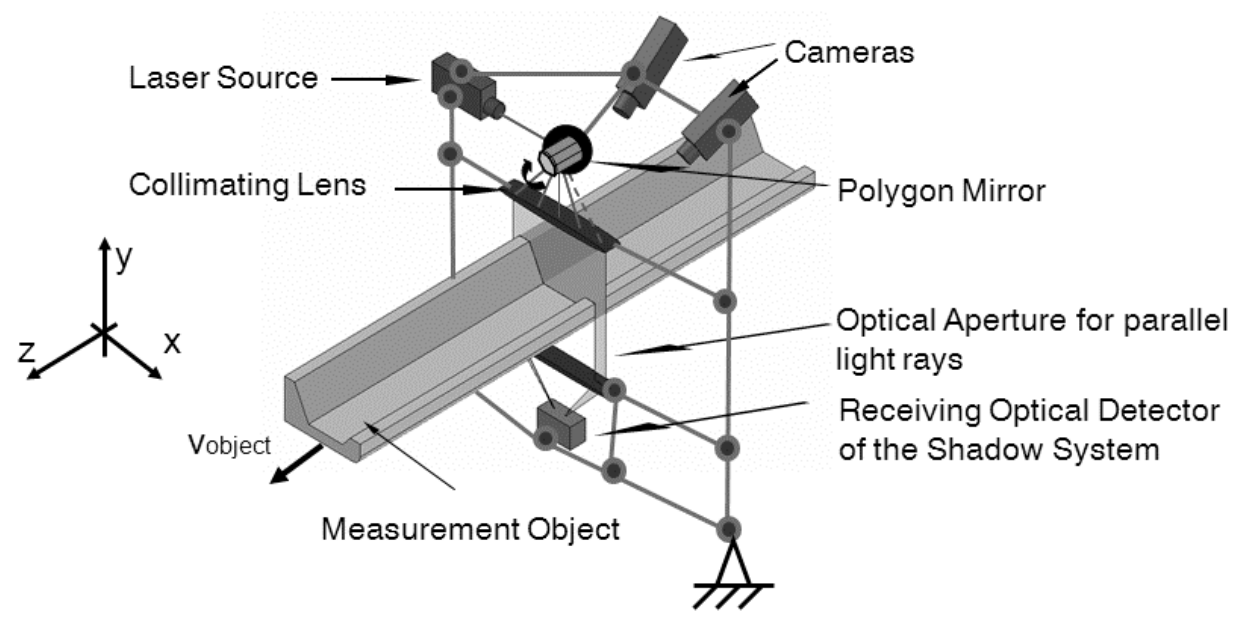

Fig. 1. Concept of the optical Multi-Sensor Measurement System.

\section{Requirements to the Measurement System}

In order to meet the requirements one has to operate with a measurement system which combines both sensor types on the one hand with more accurate results and on the other hand with higher data density. The possibility to measure concave profile zones as well has to be implemented. For the accuracy a shadow system is getting used with resolutions of down to $0.01 \mathrm{~mm}$ but it only deals with scalar length information of the boundary points of the measured profile. For measuring concave zones three light-section systems observe the profile by $360^{\circ}$ around it. Their data will be fused to one dataset and the characteristic length, roundness or other defects can be detected afterwards with a user interface including visualization. For that use suitable sensors, a flexible and stiff construction and an efficient solution for the data analysis have been built up. The parameters of the sensors must bear ambient conditions like temperature, vibration, dust, humidity and infrared radiation [2].

\subsection{Illumination}

As the illumination source a semiconductor laser is best suited because of the optical stability, fair price and at the same time less needed space and warm-up time. Blue and similarly green light (wavelengths between $420 \mathrm{~nm}$ to $530 \mathrm{~nm}$ ) are pricy and dangerous for human eyes of participant operators. The red laser (about $660 \mathrm{~nm}$ wavelength) is affordable, less dangerous and available with high power. But the vicinity in the spectrum near to the infrared light could be a problem due to the loss of contrast in the image what leads to inaccuracy and accordingly to bad measurements [3]. A performed worst case simulation brought out that red light is adequate for this application. Finally, $50 \mathrm{~mW}$ light power and an angle of the light fan of $40^{\circ}$ ensure a sharp and strong line projection despite of heat radiation. The displacement to the sensor is important for cooling practices in the shop floor later on and at the same time the width is wide enough for the device under test, see 2.4 .

\subsection{Observance of the Object}

The projected laser line must be observed stationary and has to reach accuracies to $0.1 \mathrm{~mm}$, but a camera with sensitivity, high resolution and small need of space is suited. The refresh rate must be over 20 frames per second (fps) to ensure at least one grab every $250 \mathrm{~mm}$ (the profiles extrudes with velocities up to $8 \mathrm{~m} / \mathrm{s}$ ). With respect to great light intensity a CCD-Chip with 1920 to 1080 
pixels was selected. The wide band format is suitable to measure medium distorted lines on complex profile shapes. Filters against UV and infrared radiation help to separate the disturbances. The cameras are freely adjustable in angle and distance.

\subsection{Measurement objects}

The objects are several extruded profiles from brass, aluminum and polymers. They are endless semi-finished products from $10 \mathrm{~mm}$ to $100 \mathrm{~mm}$ diameter and used in a couple of technical applications. As a result the end customer does not have to rework in the same amount to usual raw material. The profiles have temperatures of $800^{\circ} \mathrm{C}$ and move non-constantly in speed. Unknown frequencies and shocks from the process complicate the measuring place. In addition, lubricant, dust and oxide particles are constraints for the optical system in addition. In all, there are different forms to measure: H-, L-, T-, round-, hexagonal-profiles and some special types.

\subsection{Reconstruction of the part contour}

The grabbed images have to be recorded concerning the form deviation of the object. Normal triangulation works only with two angles between the object and the observer for easy reconstruction, see Fig. 2.

$$
\Delta h=\Delta l \frac{1}{\sin \beta \cdot(\cot \alpha+\cot \beta)} .
$$

On the one hand the light section systems have to be adjusted with respect to the different profiles. On the other hand, only known and steady relations of distance and angle can lead to accurate reconstruction by triangulation. In general, the reconstruction does not allow to effort much time because of real time abilities. For instance by serving with $20 \mathrm{fps}$, one measurement with all operations has obviously only $50 \mathrm{~ms}$ time to be proceeded. For this reason an extensive calibration concept had to be formulated and implemented, see 4.3 [4].

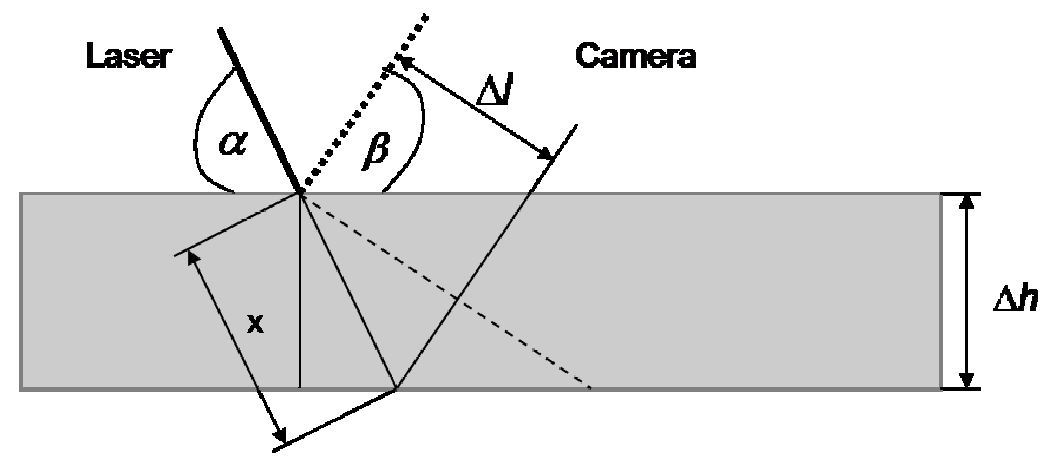

Fig. 2. Principle of the optical triangulation.

\section{Set-up of a Prototype}

The system consists of two sensor systems which have to combine the advantages of both methods optimally. For testing and evaluating the reachable performance a test stand has been constructed, see Fig. 3. The frame is made of aluminum, the light section systems - cameras and lasers - are fully adjustable around the part. 


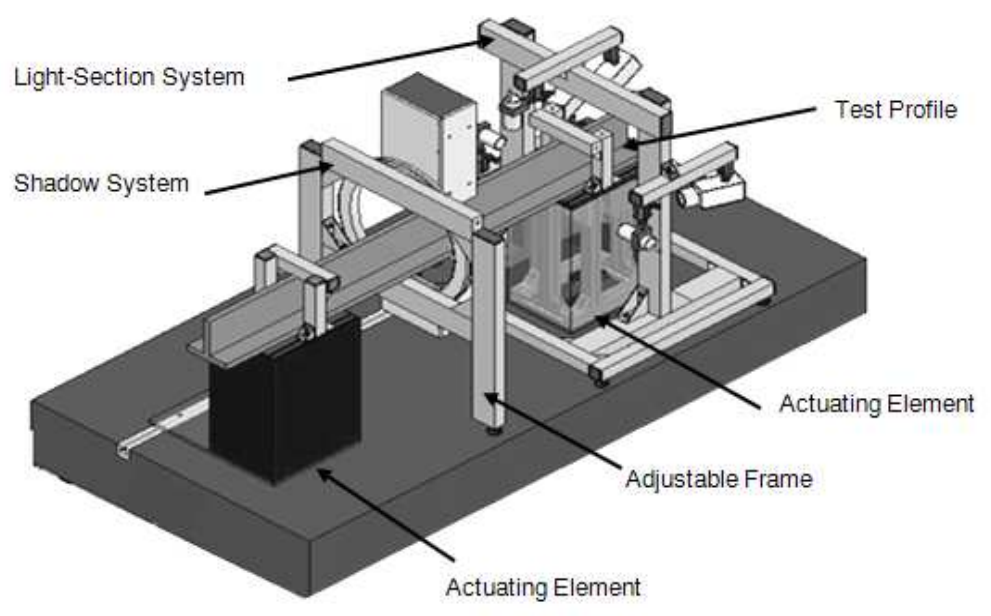

Fig. 3. Mock-up of the bi-sensorial test stand.

\subsection{Arrangement}

There are three sub-systems built in to ensure a $360^{\circ}$ view around complex-shaped or concave profiles. For this reason an adjustment concept for every profile size and type helps to install, adjust and calibrate the test stand for different sizes and types again. The distances of the several lasers, their angle, the distance of all cameras, their angle and the distance between the cameras and the lasers have to be respected [5]. To follow the parameters operatively and accurately a calibration procedure with various steps must be fulfilled, see 4.3.

\subsection{Main Elements}

Flexible knob holders allow to adjust and to fix all sensors in two rotatory and two translational degrees of freedom. The measurand can be changed easily and held or isochronally moved automatically in two dimensions by actuating elements. Linear motors with stored program control (SPC) serve with power for profile pieces up to $60 \mathrm{~kg}$ and frequencies from $0.1 \mathrm{~Hz}$ to $5 \mathrm{~Hz}$. This movement simulates the real vibrations and shocks on the shop floor. The shadow system is easily and fully rotatable $-360^{\circ}$ around the part. The shadow system can be adjusted depending on different profile types for ensuring the best view, too. A workstation with customized software conjoining flexible libraries of high-level language, ensure the image acquisition, the calibration, all calculations and the merging.

\section{Measurements}

All systems' sensors have to be motivated, images have to be read out and datasets have to be stored in a history. Afterwards the calibration, merging and reconstruction for the evaluation of form deviation are to proceed.

\subsection{Light-section-systems}

For a full view, more than one sensor pair is needed (see 3.). Three pairs are a compromise between costs, effort and the possibility of measuring complex zones. The images from every camera are triggered by a master signal, see Fig. 4a. Every picture can be sized and enhanced by contrast, Fig. 4b. The Edge Finder localizes the contrast of the laser and saves it in arrays, Fig. 4c. The three sensor pairs are installed with an optical overlap of about $10 \%$ to ensure the reconstruction to one form by algorithms based on RANSAC (Random Sample Consensus) 
perfectly. The calibration of every profile is done by a protractor for the lasers and by gauge blocks plus calibration plates for the cameras distortions and distances.

a)

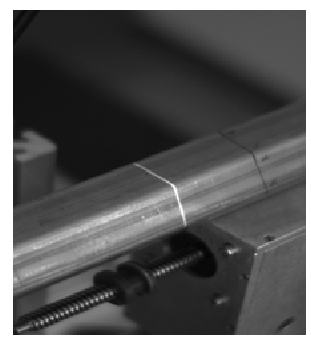

b)

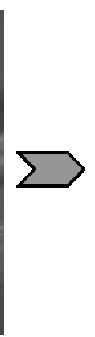

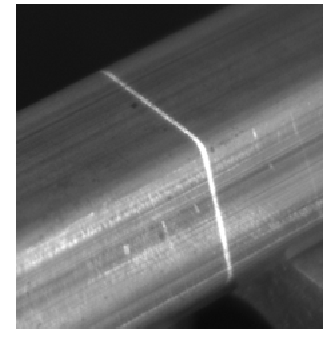

c)

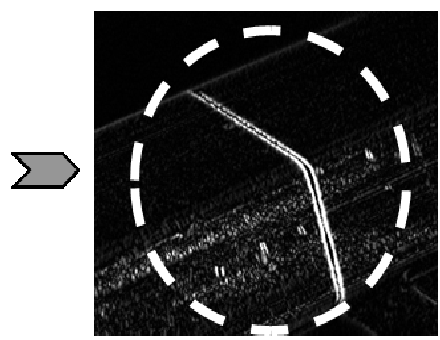

Fig. 4. Grabbed film by the light-section system under movement.

\subsection{Shadow subsystem}

The shadow system deals with scalar length information of the maximum outline of the profile by reducing the task to timing. The system has to be continuously calibrated by the accurate twopoint information. The linear calibration is done by two different sizes of gauge blocks and compensates the offset error and the pitch error. The binary information is read out directly and can be used for the full calibration and the outlines' speed of the data stream.

\subsection{Calibration of the combined system}

The calibration of all sensors demands global extrinsic coordinate systems. It has to be built together, calibrated for every type in the catalogue and aligned to one scatterplot for serving with authentic proposals of measurement uncertainty. The integration with a fluctuating point of origin has to be provided. The calibration with many degrees of freedom for the conjoined optical measurement system can be done by theodolites, gauge blocks and other special artefacts to the traceability of the unit of length, the meter.

\subsection{Datafusion - combining the results}

To combine the three datasets which are calibrated and reconstructed together, the ICPalgorithm, (iterative closest point) which is available in different variants can be used. The overlapped zones are used to straighten and align the datasets. After that, the more accurate length of the outline by the shadow system can be weighed and calculated with the result of the merged light-section point-cloud at zones near to the maximum width. Finally, the results can be analyzed and evaluated.

\section{User Interface}

The operator must easily use and analyze the reconstruction and the measurement results. He must be guided through the measurement and help has to be provided if needed to ensure stability for the process control in-line.

\subsection{Procedure of reconstructing shaped elements}

All extruding profiles must be compared with a reference which normally comes from the technical drawing. The reconstruction of the measured point-cloud with a comfortable graph and the visualization of the measured contour as well as comparing them to a reference - in due to 
evaluate the conformity or non-conformity of the part - needs an intuitive user-interface implicitly. It can be selected to which criteria shall be reconstructed. Implemented for the circles and loop structures are the Gaussian, Inscribed, Maximum, Minimum methods and - very important for many applications - the Circumscribed Maximum [6] method. Other methods provided are e.g. radius and line structures. For all visualization, calculations and experiments the ability of calculating time and accuracy must be ensured, see 5.4 and 5.5.

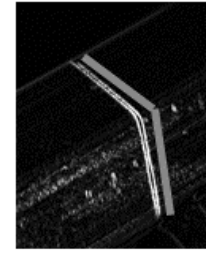

observed profile zone

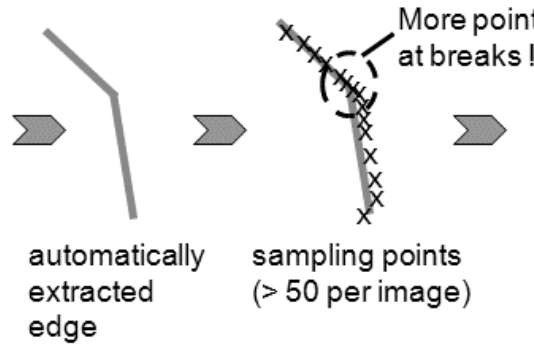

Fig. 5. Image processing and reconstruction in arrays.

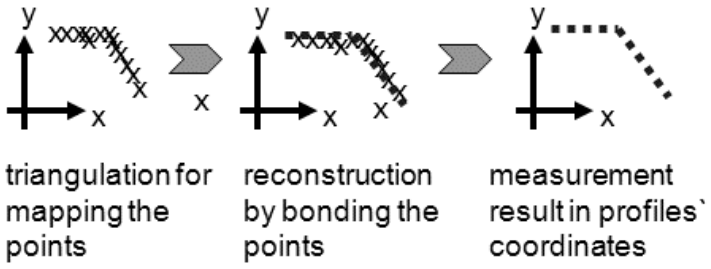

coordinates

\subsection{Evaluation of the measurement results}

The user-interface shows the reference dataset of any profile and the measured one with all calculated sampling points. Different colors help to divide into "good" or "not good" sections and to control the process performance indicators, the machine capability and the process quality. Form deviations, tolerances from length, form and position after DIN ISO 1101 can be analyzed and evaluated [7].

\subsection{Guidance to the operator}

A customized help function which blocks unknown windows and provides safety against "misuse", ignoring wrong inputs by keyboard and an ergonomic user-interface design conform to DIN ISO 9241-10 and -17 ensures intuitive and optimal usability. The operator has an easily usable limited task bar with big sized letters on the screen. For the developer a fully equipped task bar can be faded in by a hotkey with advanced usability for calibration and attendance affairs.

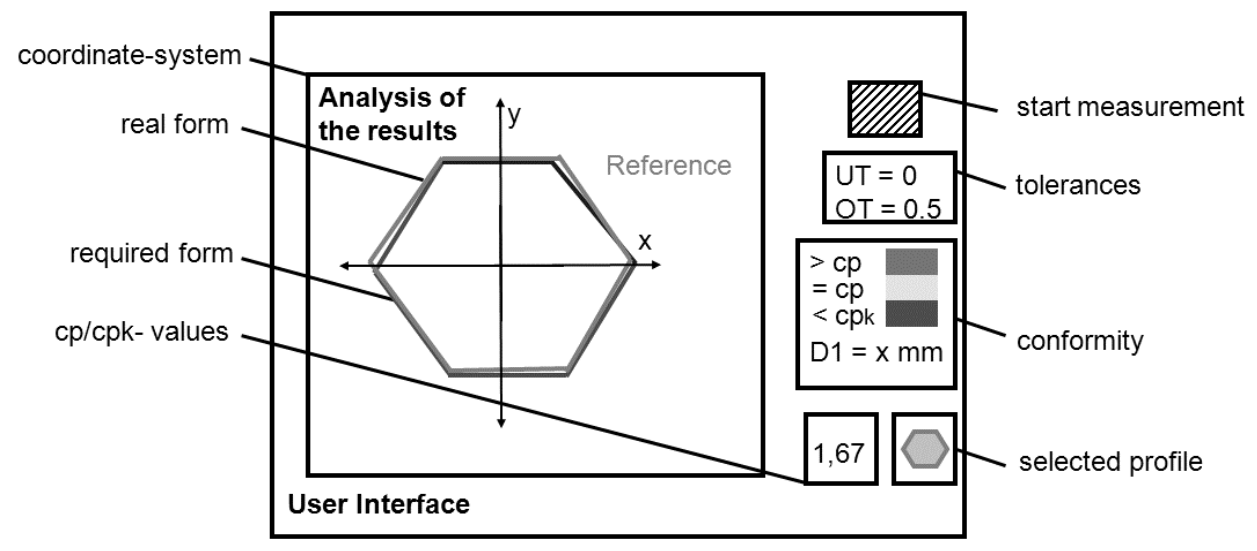

Fig. 6. User interface for the multi-sensor measurement system. 


\subsection{Calculation time}

The calculation time depends on the profile and the density of points per reconstruction [8]. Every measuring point has to be positioned at the profile by comparing with all others and collecting the distance to the normal vector of the chosen form element. E.g. the circumscribed maximum circle with $n$ points has as many possibilities $P$ :

$$
\begin{gathered}
P=(n-3) \cdot \frac{n !}{3 !(n-3) !}=(n-3) \cdot\left(\frac{n}{3}\right), \\
\stackrel{\text { (2) }}{\longrightarrow} \text { for }(n=50), P=\left(47 \cdot \frac{50}{3}\right) \approx 783 .
\end{gathered}
$$

Neglecting the most complex profile types, like $W$ or $H$, a calculation time of $30 \mathrm{~ms}$ is sufficient. The hardware-based time for image grabbing and the interface declaration are smaller than $10 \mathrm{~ms}$. In the sum of $40 \mathrm{~ms}$ (in all) finally it allows $25 \mathrm{fps}$. So, the cameras can be utilized optimally.

\subsection{Accuracy of the calculation}

The accuracy is - more than one magnitude - better than it would play a role for the agglomerated accuracy of the multi-sensorial optical measurement system. The complexity of the different calculations is very varying, so the number of reachable calculations per section is between $5^{*} 10^{3}$ up to $8^{*} 10^{4}$. The resulting deviation in maximum - in off-line tests by simulation - has been smaller than $0.01 \mathrm{~mm}$ and can be improved by more selected points for the reconstruction and analysis anytime. The accuracy and the time for calculation are competing objectives. The form elements of reconstructing straight zones, radii or pitch circles are getting less precise with less point density improving the measurement time (see 5.4). The measurement uncertainty of the optical Multi-Sensor-Measurements has to be better than $0.1 \mathrm{~mm}$ at every profile. As well at test measurements in the shop floor with outside influences, what is a topic of the current activities. Therefore the accuracy to a couple of characteristics like diameter, parallelism or perpendicularity of different profile sizes and its sections are being determined carefully. The results show that the measurement uncertainty is always better than $0.1 \mathrm{~mm}$ and as a result the calculations inaccuracy is negligible.

\section{Conclusions}

The introduced multi-sensorial measurement system consists of an optical light-section system and a shading system which are merged together to provide one measurement result. The objective to align high data rates with accuracy and the possibility to measure concave profiles has been achieved. All sensor systems are running with a PC-workstation which works with specific software. A custom-designed user-interface helps to receive robust, quantitatively repeatable results from data points reconstructed measurement. Finally a $360^{\circ}$ view as well of complex profiles is enabled and analyzes the profiles optimally in-line. The method operates in spite of rough constraints in the shop floor. The evaluation of length, form and position compared to a reference is directly possible and ergonomically usable, to select parts in "conform" and "non-conform" class at any time, with a frequency of up to 25 times per second and a measurement uncertainty better than $0.1 \mathrm{~mm}$. The next steps are to fine the calibration of the sensors, giving quantitative proposals to the measurement uncertainty, optimizing the measurement results and the measurement process and improving the juncture between hardware and software for even faster results. After that, the optical multi-sensorial measurement system 
can be optimized and can be installed to the manufacturing process where many influences will be critical. It assists to monitor the process in real-time better than before what leads to better product quality and less rework at the end customer of semi-finished products.

\section{Acknowledgment}

This research was founded by the Bavarian Research Foundation (BFS).

\section{References}

[1] Bauer, N. (2003). Compendium to image-guided Sensor Technology. Leitfaden zur bildgebenden Sensortechnik. Fraunhofer, Erlangen.

[2] Trostmann, E. (2003). Compendium to Fundamentals and Applications of optical 3D-Metrology. Leitfaden zu Grundlagen und Anwendungen der optischen 3-D Messtechnik. Fraunhofer, Erlangen.

[3] Pfeifer, N., Briese, C. (2007). Laser Scanning - Principles and Applications. Moskau.

[4] Wagner, T. (2000). Automated Configuration of Image Processing Units. Automatische Konfiguration von Bildverarbeitungssystemen. Erlangen-Nuremberg.

[5] Hoffmann, J. (2001). User Guide of Metrology. Handbuch der Messtechnik. Munich: Hanser.

[6] Weckenmann, A., Eitzert, H., Weber, H. (1992). Coordinate Metrology evaluating function-oriented. Conference on Microtechnology. VDI.

[7] Weckenmann, A., Eitzert, H., Garmer, M., Weber, H. (1995). Functionality-oriented evaluation and sampling strategy in coordinate metrology. University of Federal Armed Forces, Hamburg.

[8] Maresch, T. (2006). Mathematics, Conjunction of 2-D and 3-D Pointclouds. Mathematik, Verknüpfung von $2 D$ - und $3 D$-Punktwolken. Giessen, Dissertation. 